\title{
$X L$. On the accuracy of focus necessary for sensibly perfect definition
}

\section{Lord Rayleigh F.R.S.}

To cite this article: Lord Rayleigh F.R.S. (1885) XL. On the accuracy of focus necessary for sensibly perfect definition, Philosophical Magazine Series 5, 20:125, 354-358, DOI: $10.1080 / 14786448508627768$

To link to this article: http://dx.doi.org/10.1080/14786448508627768

曲 Published online: 29 Apr 2009.

Submit your article to this journal $\sqsubset \pi$

Џ Article views: 5

Q View related articles $₫$ 
TABLE VI.

\begin{tabular}{|c|c|c|c|}
\hline Date. & $\begin{array}{l}\text { Standard of } \\
\text { Comparison. }\end{array}$ & B.A.U. in M. & M. in B.A.U. \\
\hline April 9 . & Flat. & $1 \cdot 04814$ & .95407 \\
\hline, $15 \ldots \ldots \ldots \ldots \ldots$ & 54 . & $1 \cdot 04806$ & $.95+13$ \\
\hline$"$, & Flat. & $1 \cdot 04791$ & $\cdot 95427$ \\
\hline $16 \ldots \ldots \ldots \ldots$ & Flat. & $1 \cdot 04820$ & .95402 \\
\hline$"$, & 里 54 & $1 \cdot 04811$ & 95410 \\
\hline , $17 \ldots$ & 䨤 54 and 20 . & $1 \cdot 04801$ & $.95 \pm 19$ \\
\hline 17 & Flat and 20. & 1.04821 & .95399 \\
\hline " $, \quad, \ldots \ldots \ldots \ldots . . . . .$. & Flat and 20. & $1 \cdot 04797$ & $-95+22$ \\
\hline \multicolumn{2}{|l|}{ Mean } & $1 \cdot 04808$ & .95412 \\
\hline
\end{tabular}

These numbers, it will be seen, agree with those found by Lord Rayleigh and Isorenz, and differ greatly from Strecker's values.

They represent the results of the comparison of a Germansilver copy of Strecker's tubes with the B.A. units, including the actual coil employed by Strecker, 电 No. 54; while Strecker's numbers give the result of the comparison of his tubes directly with No. 54.

I have not been able to explain the discrepancy. Herr Strecker suggests in a letter to me on the subject, that the resistance of his coil No. 13 has changed somewhat in the year which has elapsed since his comparisons were made. At present I am in correspondence with Prof. F. Kohlrausch, of Würzburg, in whose laboratory Strecker's experiments were conducted, and hope to obtain another copy of Strecker's tubes, or at any rate to have another comparison made between his tubes and the B.A. standards.

XL. On the Accuracy of Focus necessary for Sensibly Perfect Definition. By Lord RAYLEIGH, F.R.S.*

T $N$ my "Investigations in Optics" $\dagger \mathrm{I}$ have examined the eftect upon definition of small disturbances of the wavesurfaces from their proper forms. It follows, for instance,

* Communicated by the Author.

$\dagger$ Phil. Mag. $1879 \& 1880$. 
that the aberration of a plano-convex lens focusing parallel rays of homogeneous light is unimportant, so long as the fourth power of the angular semi-aperture does not exceed the ratio of the wave-length to the focal distance $\left[\alpha^{4}<(\lambda / f)\right]$, a condition satisfied by a lens of 3 feet focus, provided that the aperture be less than 2 inches. I propose at present to apply similar principles to the question of focusing.

The most convenient point of view is that explained ${ }^{*}$ for calculating the focal length of lenses. If the lens $A B$ converges parallel rays to a focus at $F$, the retardation of the

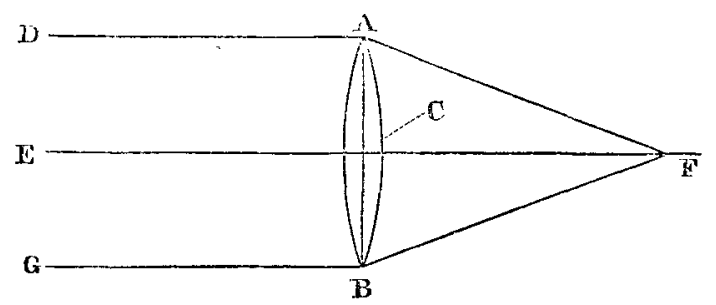

central ray $E F$, duo to the substitution of a thickness $t$ of glass for air, is $(\mu-1) t$; and this must be equal to the retardation of the extreme rays passing the (sharp) edge of the lens, i. e. $\mathrm{AF}-\mathrm{CF}$. Thus, if $\mathrm{AC}=y, \mathrm{FC}=f$,

$$
(\mu-1) t=\sqrt{ }\left(f^{2}+y^{2}\right)-f=\frac{1}{2} \frac{y^{2}}{f}, \quad . \quad . \quad \text {. }
$$

approximately, which gives the focal length in terms of the semi-aperture and the "thickness" of the lens.

If we suppose that $\mu$ varies,

$$
\delta \mu . t=-\frac{1}{2} \frac{y^{2}}{f^{2}} \delta f, \quad \cdot \quad \cdot \quad \cdot \quad .
$$

giving the change of focus required to compensate the change of $\mu$. Let us, however, inquire what is the state of things at the old focus. The secondary rays from the extreme boundary of the lens arrive with the same phase as before the change of index; but the central ray undergoes a relative retardation amounting to $\delta \mu . t$. This quantity tells us the discrepancy of phase; and we know that if it is less than $\frac{1}{2} \lambda$, the agreement of phase is still good enough to give nearly perfect definition. Hence from (2) we see that a displacement $\delta f$ from the true focus will not impair definition, provided

$$
\delta f<\frac{f^{2} \lambda}{y^{2}} \cdot \quad \cdot \quad \cdot \quad \cdot \quad \cdot .
$$

* Loc. cit. p. 480.

$2 \mathrm{C}_{2}$ 
It appears that the linear accuracy required is the same whatever the absolute aperture of the object-glass may be, provided that the ratio of aperture to focal length be preserved.

In some trials that I have made the diameter of the objectglass was $1 \frac{1}{8}$ inch, and the focal length 12 inches. Taking $\lambda=\frac{1}{40,000}$ inch, we get from (3)

$$
\delta f<\cdot 0115 \mathrm{inch},
$$

a result which corresponded very well with observation. The instruments employed were the collimator and telescope of a spectrometer, the object under examination being a slit backed with a soda-flame. A high-power eye-piece was used, and the telescope was adjusted until the edge of the slit and the wire in the eye-piece were seen well defined together. The instrument was unprovided with an easy focusing motion, so that it was not possible to try backwards and forwards conveniently. In this way the setting corresponded more closely to the suppositions of theory than if it were the result of comparisons between appearances at equal distances within and without the point chosen. It will be understood that there is no theoretical limit to the accuracy with which a focal point may be ultimately determined, if the lenses are good, and observations are multiplied with suitable precautions to avoid asymmetry.

In ten settings the extreme difference was only $\cdot 02$ inch, showing that a displacement of 01 inch from the true focal point was just recognizable.

By using various coloured flames, or by throwing a spectrum upon the slit of the apparatus, we may determine the focal length for different kinds of light. With proper achromatic lenses the differences should be pretty small, the minimum focal length corresponding to the yellow-green rays. It so happens that my instrument is far from properly compensated, and gives a fair primary spectrum, so that the difference of focus for yellow and green is very easily recognized. In the case of a single lens this method would give the dispersive power of the glass with fair accuracy. By comparison with the theory of the resolving power of prisms, we see that the dispersion is about as favourably determined with a lens as with a prism of equal thickness. In either case a change of index such that $\delta \mu . t=\frac{1}{2} \lambda$ leaves the phase agreement nearly unaltered at the original points; but in other respects the circumstances are probably rather more favourable in the case of the prism.

It is generally considered that the most accurate way of 
focusing a small telescope is to move the eye across the eyepiece, altering the adjustment until there seems to be no relative motion of object and cross wires. I have tried this plan in an improved form in order to see whether a higher degree of accuracy of adjustment was really attainable, although theory seemed to show that no great advance was to be looked for. A heavy pendulum, executing complete vibrations in about two seconds, was fitted up in front of the telescope, and carried with it a screen perforated by a slit. The width of the slit was about a quarter of the entire aperture, and the oscillations were at first of such amplitude as just to bring the extreme edges of the lens into play. In the earlier experiments the slit of the collimator was backed by the clouds, a piece of green glass being interposed. This was before I had discovered the remarkably unachromatic character of the instruments, and I was puzzled to interpret the appearances presented. On one side of the focus the relative motion of the image was (as it should be) in the same direction as that of the pendulum, and on the other side in the opposite direction; but the transition was not well defined, and the image executed evolutions very visible to the observer, who at the same time was not able to describe them as swinging in one direction or the other. The effect upon the eye was remarkably unpleasant and fatiguing to watch; it disappeared when recourse was had to sodium light, and doubtless depended upon the variation of quality in the light. It may be noticed that spherical aberration would show itself by a swinging of the image in a period half that of the pendulum.

With the soda-flame the adjustment to focus by getting rid of the swinging motion was pretty accurate ; but not much advantage was gained in comparison with a setting by simple inspection under full aperture. As before, the extreme difference in a set of ten was about 02 inch.

The substitution of white for monochromatic light was instructive. In either extreme position of the oscillating slit the light was seen to be spread into a spectrum of moderate length, the blue and red being interchanged after each half period. Under these circumstances the cross wires can be made to maintain their position in that part of the spectrum only for which the telescope is focused. If, for example, it be the green of the spectrum, we may bring the cross wires to this position when the pendulum is at rest, and then, in spite of the oscillation, the position will be maintained. If, without altering the focus, we move the cross wires to another part of the spectrum, then, when the pendulum oscillates, the 
wires will be seen on a different part of the spectrum after each half period. In order to fix the new part of the spectrum upon the cross wires, a clange of focus is demanded. 'This experiment would hardly succeed with properly compensated object-glasses, but it could be imitated with the aid of single lenses.

XLI. On an Improved Apparatus for Christiansen's Experiment. By Lord RAYLEIGH, F.R.S.*

THE very beautiful experiment in question, described by 1 C. Christiansen in Wiedemann's Annalen for November 1884 , consists in immersing glass-powder in a mixture of benzole and bisulphide of carbon of such proportions that for one part of the spectrum the indices of the solid and of the fluid are the same. Being interested in this subject from having employed the same principle for a direct-vision spectroscope (Phil. Mag. January 1880, p. 53), I have repeated Christiansen's experiment in a somewhat improved form, which it may be worth while briefly to describe, as the matter is one of great optical interest.

I must premise that the beauty of the effect depends upon the correspondence of index being limited to one part of the spectrum. Rays lying within a very narrow range of refrangibility traverse the mixture freely, but the neighbouring rays are scattered laterally much as in passing ground glass. Two complementary colours are therefore exhibited, one by direct, and the other by oblique, light. In order to see these to advantage, there should not be much diffused illumination, otherwise the directly transmitted moncchromatic light is liable to be greatly diluted. The prettiest colours are obtained when the undisturbed rays are from the green; but the greatest general transparency corresponds to a lower point in the spectrum.

The improvement referred to relates merely to the use of a flat-sided bottle to contain the preparation. In order to get a satisfactory result it is necessary that the sides of the containing vessel be pretty good optically. This condition may be satisfied with a built-up cell, but on account of the difficulty of finding a suitable cement, it is rarely that such cells remain in good order for any length of time. It occurred to me that a bottle might be made to answer the purpose, provided the precaution were taken of using the same kind of glass for the bottle and for the powder. The outer sur-

* Communicated by the Author. 